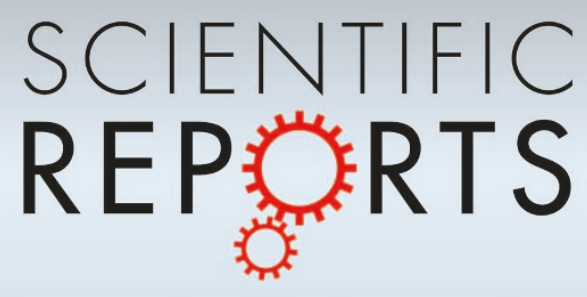

OPEN

SUBJECT AREAS:

QUANTUM HALL

MAGNETIC PROPERTIES AND

MATERIALS

Received

28 July 2014

Accepted

15 August 2014

Published

10 September 2014

Correspondence and requests for materials should be addressed to

D.N.S. (donna.

sheng1@csun.edu)

\title{
Emergent Chiral Spin Liquid: Fractional Quantum Hall Effect in a Kagome Heisenberg Model
}

\author{
Shou-Shu Gong, Wei Zhu \& D. N. Sheng
}

Department of Physics and Astronomy, California State University, Northridge, California 91330, USA.

The fractional quantum Hall effect (FQHE) realized in two-dimensional electron systems under a magnetic field is one of the most remarkable discoveries in condensed matter physics. Interestingly, it has been proposed that FQHE can also emerge in time-reversal invariant spin systems, known as the chiral spin liquid (CSL) characterized by the topological order and the emerging of the fractionalized quasiparticles. A CSL can naturally lead to the exotic superconductivity originating from the condense of anyonic quasiparticles. Although CSL was highly sought after for more than twenty years, it had never been found in a spin isotropic Heisenberg model or related materials. By developing a density-matrix renormalization group based method for adiabatically inserting flux, we discover a FQHE in a spin- $\frac{1}{2}$ isotropic kagome Heisenberg model. We identify this FQHE state as the long-sought CSL with a uniform chiral order spontaneously breaking time reversal symmetry, which is uniquely characterized by the half-integer quantized topological Chern number protected by a robust excitation gap. The CSL is found to be at the neighbor of the previously identified $Z_{2}$ spin liquid, which may lead to an exotic quantum phase transition between two gapped topological spin liquids.

$\mathrm{T}$ he experimentally discovered fractional quantum Hall effect $(\mathrm{FQHE})^{1-3}$ is the first demonstration of topological order and fractional (anyonic) statistics ${ }^{4-8}$ realized in two-dimensional electronic systems under a magnetic field breaking time-reversal symmetry (TRS). A related new state of matter with fractionalized quasiparticle excitations is the topological quantum spin liquid (QSL) emerging in frustrated magnetic systems $s^{9-18}$. Such spin systems, related to strongly correlated Mott materials and holding the clue to the unconventional superconductivity in doped systems, are of fundamental importance to the condensed matter field ${ }^{9,10,18-20}$. To understand the emergent physics of frustrated magnetic systems, where spins escape from the conventional fate of developing symmetry broken ordering, the concept of QSL with the fractionalized quasiparticles was established ${ }^{8,10,14}$. Experimental candidates for such a new state of matter are identified including kagome antiferromagnets ${ }^{21-23}$ and triangular organic compounds ${ }^{24-26}$. The simplest QSL with TRS is the gapped $Z_{2}$ spin liquid, which possesses the $Z_{2}$ topological order and fractionalized spinon and vison quasiparticle excitations ${ }^{8,14}$. The $Z_{2}$ QSL is identified as an example of the resonating valence-bond liquid state, which was first proposed by Anderson ${ }^{10}$. Although explicitly demonstrated in contrived theoretical systems ${ }^{11,13,16,17}$, the searching of the gapped QSL in realistic Heisenberg models has always attracted much attention over the last twenty years. The primary example is the recent discovered gapped $Z_{2}$ QSL for kagome Heisenberg model (KHM) with the dominant nearest neighbor (NN) interactions based on the density-matrix renormalization group (DMRG) simulations $\mathrm{s}^{27-30}$.

Another class of QSL with fractionalized quasiparticles obeying fractional (anyonic) statistics is chiral spin liquid (CSL) $)^{31-35}$, which breaks TRS and parity symmetry while preserves other lattice and spin rotational symmetries. Kalmeyer and Laughlin ${ }^{31}$ first proposed that, in a time-reversal invariant spin system with geometry frustration, one can realize a $v=1 / 2 \mathrm{FQHE}$ as a CSL state ${ }^{32}$ through mapping the frustrated in-plane exchange interactions to the uniform magnetic field. A CSL is also considered to be a simple way in which frustrated spin systems develop topological order through statistics transformation to cancel out the frustration ${ }^{32,33}$. The CSL may also lead to the exotic anyon superconductivity with doping holes into such systems $\mathrm{s}^{32,33}$. The existence of CSL through spontaneously TRS breaking has been demonstrated in a Kitaev model on a decorated honeycomb lattice with contrived anisotropic spin interactions ${ }^{36}$ and most recently in a spin anisotropic kagome model ${ }^{37}$. Interestingly, based on the classical and Schwinger boson mean-field analyses, QSLs with different chiral spin orders have been suggested for extended $\mathrm{KHM}^{38,39}$. Other theoretical studies show that one can also induce a CSL 
state through adding multi-spin TRS breaking chiral interactions ${ }^{40-43}$. Although CSL has been explored for more than twenty years $^{31-35,38,43,44}$, the accurate $\mathrm{DMRG}^{27-30}$ and variational Monte Carlo $^{45}$ studies on various frustrated Heisenberg models often lead to the conventional ordered phases or TRS preserving $Z_{2}$ and $U(1)$ QSLs. The simple concept of realizing CSLs through spontaneously breaking TRS and statistics transformation ${ }^{32,33}$ remains illusive in realistic frustrated magnetic systems.

In this article, we report a new theoretical discovery of the CSL in an extended spin- $\frac{1}{2}$ KHM based on the state of art DMRG simulations ${ }^{46,47}$. As illustrated in the inset of Fig. 1(a), the system has the NN coupling $J=1$ as energy scale, as well as the second and third NN couplings $J^{\prime}$ inside each hexagon of the kagome lattice, described by the following Hamiltonian ${ }^{16,38}$ :

$$
H=J \sum_{\langle i, j\rangle} S_{i} \cdot S_{j}+J^{\prime} \sum_{\langle\langle i, j\rangle\rangle} S_{i} \cdot S_{j}+J^{\prime} \sum_{\langle\langle\langle i, j\rangle\rangle\rangle} S_{i} \cdot S_{j} .
$$

We perform the numerical flux insertion simulations on cylinder systems based on the newly developed adiabatical DMRG to detect the topological Chern number, which uniquely characterizes the chiral spin liquid. We have fully established a robust $v=1 / 2$ FQHE state for $0.1 \lesssim J^{\prime} \lesssim 0.7$ by observing the half-integer quantized topological Chern number protected by a robust excitation gap, the
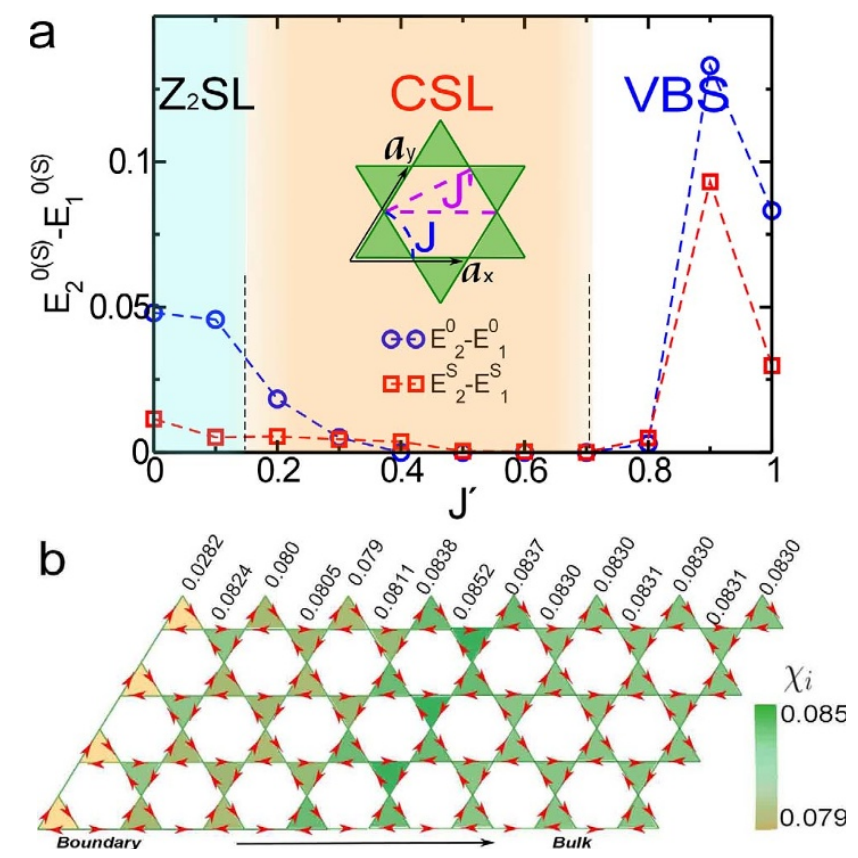

Figure $1 \mid$ Phase diagram of the spin- $\frac{1}{2} J-J^{\prime}$ antiferromagnetic kagome Heisenberg model (KHM). We set the NN coupling $J=1$, and the second and third NN couplings as $J^{\prime}$. In DMRG simulations, we study cylinders with lengths $L_{x}$ and $L_{y}$ (in unit cells) along the $x$ and $y$ (the tilt lattice vector) directions, respectively. We also label our system by the total number of sites $N=3 \times L_{x} \times L_{y}$. (a) The energy differences between the two lowest states $E_{2}^{0}-E_{1}^{0}\left(E_{2}^{S}-E_{1}^{S}\right)$ in two different topological (vacuum and S-) sectors are shown for a $N=3 \times 24 \times 4$ cylinder at different $J^{\prime}$, which reveal the four degenerating ground states in two sectors for a range of parameter $0.2 \lesssim J^{\prime} \lesssim 0.7$. (b) We illustrate the uniform positive chiral order $\left\langle\chi_{i}\right\rangle=\left\langle S_{i_{1}} \cdot\left(S_{i_{2}} \times S_{i_{3}}\right)\right\rangle\left(i_{1}, i_{2}, i_{3} \in \Delta_{i}\left(\nabla_{i}\right)\right)$ on a $N=3 \times 24 \times 4$ cylinder with $J^{\prime}=0.5$ measured from the MES $\left|\tilde{\psi}_{L}^{0}\right\rangle$ in the vacuum sector (identified in Fig. 3), which breaks the TRS and parity symmetry. The CSL phase is characterized by the long-range chiral correlations and a fractionally quantized $C=1 / 2$ Chern number, which identifies the state as the Laughlin $v=1 / 2$ FQHE emerging in the $J-J^{\prime}$ KHM. degenerate ground states, and the uniform chiral order spontaneously breaking TRS.

\section{Results}

Phase diagram. Our main findings are summarized in the phase diagram Fig. 1(a). With the turn on of a positive $J^{\prime}$, we find a robust CSL phase in the region of $0.1 \lesssim J^{\prime} \lesssim 0.7$. We design and perform the Laughlin flux insertion numerical experiment through developing an adiabatic DMRG, which inserts flux and obtains the ground state for each flux. The adiabatic DMRG allows us to obtain the topological Chern number ${ }^{3,34}$, which characterizes the topological nature of the quantum phase. Our simulation experiment shows that the CSL is characterized by a fractionally quantized Chern number $C=1 / 2$, which is a "smoking gun" evidence of the emergent $v=1 / 2$ Laughlin FQHE state ${ }^{31}$ in the frustrated KHM. The CSL phase is also characterized by a four-fold degeneracy in two topological sectors. In each sector, there is a double degeneracy representing the two sets of CSL states with opposite chiralities. The near uniform chiral order measured for a state spontaneously breaking TRS is illustrated in Fig. 1(b). We also establish that the CSL is neighboring with the $Z_{2}$ QSL previously found ${ }^{27-30}$ at $J^{\prime}=0$, while the transition region appears to be under strong influence of the nonuniform Berry curvature resulting from gauge field, which may provide new insights to many puzzles regarding theoretical ${ }^{27-30}$ and experimental findings ${ }^{18,21-23}$ for kagome antiferromagnets.

Fractional quantization of topological number. To uncover the full topological nature of the phase at large system scale, we perform the flux inserting simulation based on the adiabatic DMRG. For conventional FQHE systems, a quantized net charge transfer would appear as $\Delta N=C$ from one edge of the sample to the other edge after inserting one period of flux $\theta=0 \rightarrow 2 \pi$, corresponding to a nonzero fractionally quantized topological invariant Chern number $C^{34}$, which is $C=1 / 2$ for the $v=1 / 2$ bosonic Laughlin state.

By adiabatically inserting the flux $\theta$ in our DMRG experiment, we study the evolution of the local magnetization $\left\langle S_{x, y}^{z}\right\rangle$, which is the spin- $z$ average of the ground state at a local lattice site $R_{i}=(x, y)$. With the increase of $\theta$, we measure the corresponding spin accumulations of each ground state at $\theta=j \pi / 2$ ( $j$ is an integer). One example with $\theta=2 \pi$ is shown in Fig. 2(a). We find nonzero magnetization starting to build up at the left and right edges of cylinder, which grows monotonically with the growing of $\theta$ as shown in Fig. 2(b). Since our system has total spin conservation, the net spin-z transfer $\left.\Delta S^{z}\right|_{\text {edge }}$ (which is the total magnetization around the right edge of the system) is equivalent to the pumping of the hardcore bosons from the left edge to the right edge without going through the bulk. In Fig. 2(c), we show the net spin transfer $\left.\Delta S^{z}\right|_{\text {edge }}$ as a function of $\theta$. A near linear spin pump is being realized in this chiral spin state, which is exactly quantized as $\left.\Delta S^{z}\right|_{\text {edge }}=0.5$ at $\theta=2 \pi$. From the fundamental correspondence between edge spin transfer and bulk Chern number ${ }^{48}$, we identify the bulk Chern number of the system as $C=1 / 2$, fully characterizing the state as the Kalmeyer-Laughlin $\mathrm{CSL}^{31}$ of $v=1 / 2$ FQHE. Physically, the pumping in FQHE system is achieved through the adiabatical rotation of the basis states of the many-body wavefunction, which can be viewed as a non-local operation by developing a "spinon" line in the cylinder. We find the entanglement spectrum of the spinon sector obtained here by inserting $2 \pi$ flux is identical to the one of the S-sector shown below in Fig. 3(b) obtained through pinning. With further increasing the flux to $\theta=4 \pi$, the net spin transfer $\left.\Delta S^{z}\right|_{\text {edge }}=1.0$, where the system evolves back to the vacuum sector. These observations fully establish the bosonic $v=1 / 2 \mathrm{FQHE}$ emerging in the $J-J^{\prime}$ KHM. While the Chern number simulations characterize the ground state as the long-sought CSL, we will further measure the topological degeneracy, chiral correlations, topological entanglement entropy, and modular matrix to demonstrate the full nature of the topological state in our time-reversal invariant system. 
a
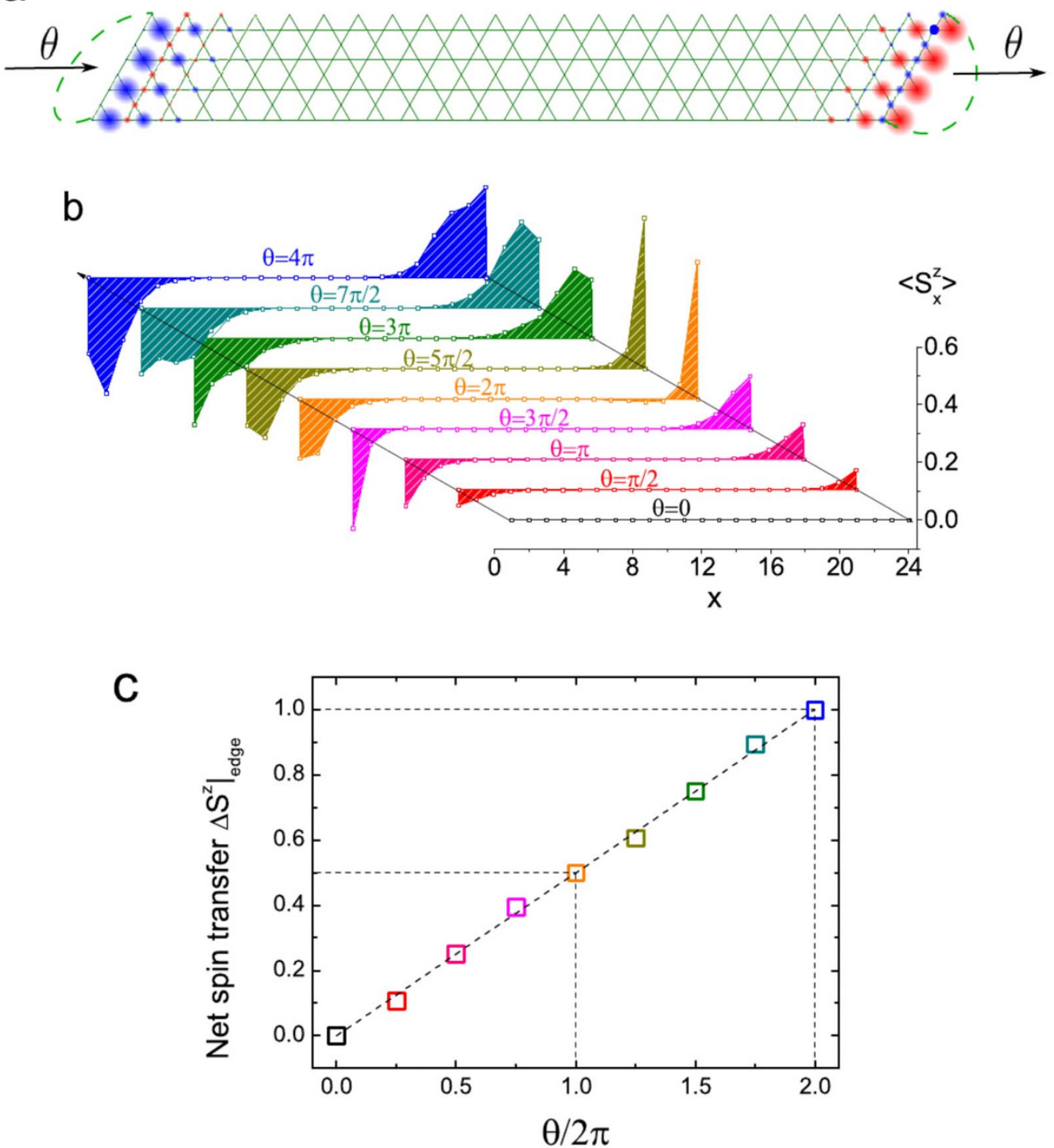

Figure $2 \mid$ Laughlin flux insertion Gedanken experiment and fractionalized Chern number $C=1 / 2$ for CSL. (a) Real-space configuration of the spin magnetization $\left\langle S_{x, y}^{z}\right\rangle$ at position $R_{i}=(x, y)$ after adiabatically inserting a quantized flux $\theta=2 \pi$. The area of the circle is proportional to the amplitude of $\left\langle S_{x, y}^{z}\right\rangle$. The red (blue) color represents the positive (negative) $\left\langle S_{x, y}^{z}\right\rangle$. (b) Real-space configuration of the accumulated spin magnetization

$\left\langle S_{x}^{z}\right\rangle=\sum_{y}\left\langle S_{x, y}^{z}\right\rangle$ (the summation is over all the $3 L_{y}$ sites in each column $x$ ) with increasing flux $\theta$. Clearly, we see a net spin-z accumulating in the right edge of the sample, which is equivalent to the transfer of hardcore bosons (the hardcore boson number $n_{i}$ is related to the on-site $S_{i}^{z}$ as $n_{i}=S_{i}^{z}+1 / 2$ ) being pumped from the left edge to the right edge without going through the bulk. So this simulation experiment reveals a quantum Hall system with a nonzero Hall conductance, while the bulk is gapped. (c) Net spin transfer $\left.\Delta S^{z}\right|_{\text {edge }}$ to the right edge of the cylinder as a function of $\theta$. From the net spin transfer in one period of flux $\theta=0 \rightarrow 2 \pi$, we obtain the exact fractionally quantized Chern number $C=\left.\Delta S^{z}\right|_{\text {edge }}=1 / 2$. The results are demonstrated for a $3 \times 24 \times 4$ cylinder at $J^{\prime}=0.5$ using the $U(1)$ DMRG with keeping up to 5000 states. Similar results are obtained for all the states within the CSL phase.

Low-energy spectrum and topological degeneracy. The KalmeyerLaughlin CSL has two-fold topological ground-state degeneracy, and the spontaneously TRS breaking for such a time-reversal invariant system must have an additional double degeneracy in each topological sector. On cylinder geometry, one can control the boundary condition near the cylinder edges to target into different topological sectors ${ }^{27,49}$, which we denote as the vacuum and S-sectors, respectively. By using this technique in DMRG, we find the two lowest-energy states in each sector whose energy differences $E_{2}^{0}-E_{1}^{0}$ and $E_{2}^{S}-E_{1}^{S}$ drop to small values for $0.2 \lesssim J^{\prime} \lesssim 0.7$. One example is shown in Fig. 1(a) for a cylinder with $L_{x}=24$ and $L_{y}=$ 4. Importantly, the degenerating states $\left|\psi_{1,2}^{0}\right\rangle\left(\left|\psi_{1,2}^{S}\right\rangle\right)$ in each topological sector also have near identical entanglement spectra.
The double degeneracy of entanglement spectrum for the ground states $\left|\psi_{1}^{0(S)}\right\rangle$ is explicitly shown using two different symbols (line and circle) in Figs. 3(a) and 3(b). These observations are consistent with the spontaneously TRS breaking double degeneracy. We also find the ground-state energies between the two sectors are degenerate $\left(E_{1}^{S} / N-E_{1}^{0} / N=0.00001\right.$ for $J^{\prime}=0.5$ at $\left.L_{y}=4\right)$, which, combined with the distinct entanglement spectra ${ }^{50}$ as shown in Figs. 3(a) and 3(b) of the two sectors, establish the topological degeneracy for these two sectors in the intermediate phase. By searching for other low energy excited states from both DMRG and exact diagonalization (ED), we exclude that there are other distinct topological degenerating sectors for the intermediate region, while a lot more lower energy states appear near $J^{\prime}=0$. 

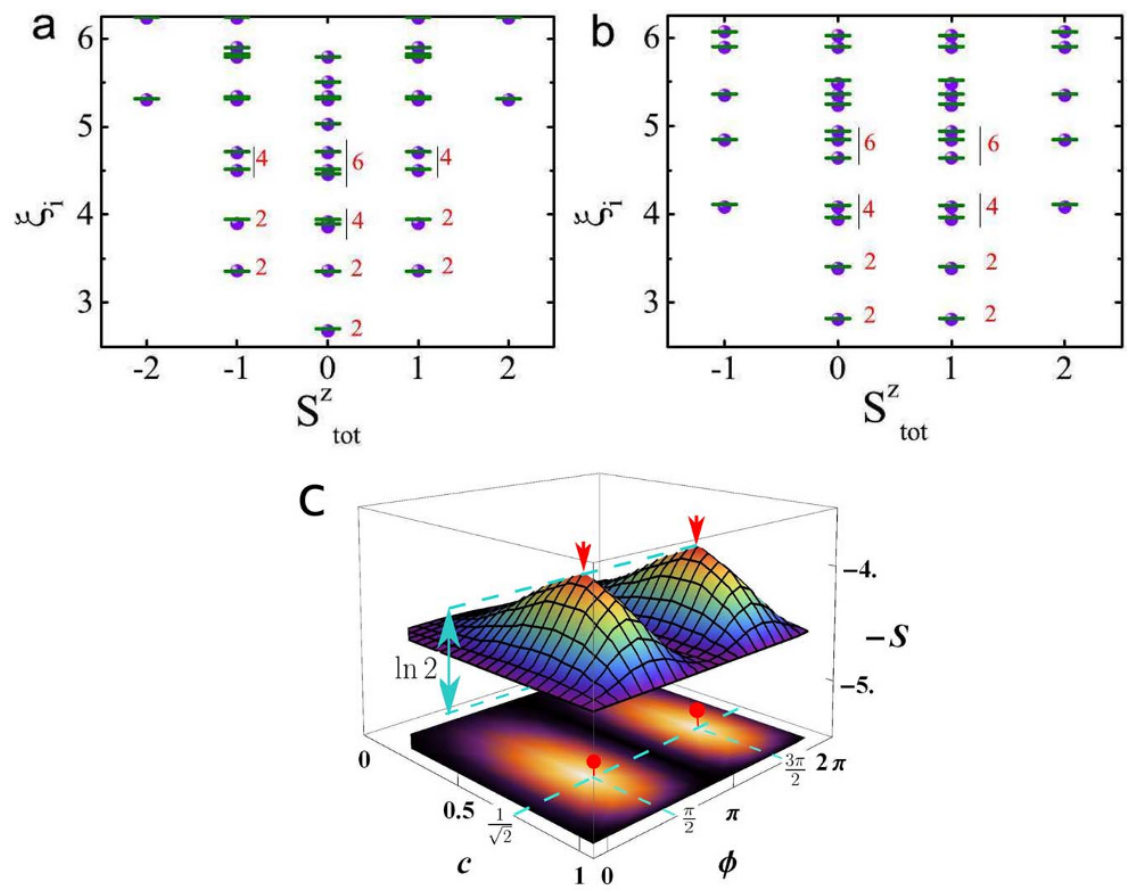

Figure $3 \mid$ Entanglement spectra characterization and MESs for the TRS broken phase. (a) and (b) are the entanglement spectra of the ground states in the vacuum $\left(\left|\psi_{1}^{0}\right\rangle\right)$ and S- $\left(\left|\psi_{1}^{S}\right\rangle\right)$ sectors, respectively. $\xi_{i}=-\ln \lambda_{i}$ with $\lambda_{i}$ the eigenvalues of reduced density matrix. The two degenerate states $\left|\psi_{1,2}^{0}\right\rangle$ $\left(\left|\psi_{1,2}^{S}\right\rangle\right)$ have exactly the same spectra. These states are real wavefunctions consistent with the TRS of the model Hamiltonian. The lines and circles with the near identical $\xi_{i}$ denote the double degeneracy indicating that each of these low-energy states is a maximum entropy state. The numbers $2,2,4,6$ label the near degenerating pattern for the low-lying entanglement spectra which are doubled from what one would expect for a Laughlin FQHE state from conformal field theory. (c) Entanglement entropy profile $-S$ of the general superposition state in the vacuum sector $\left|\tilde{\psi}^{0}\right\rangle=c\left|\psi_{1}^{0}\right\rangle+\sqrt{1-c^{2}} e^{i \phi}\left|\psi_{2}^{0}\right\rangle$. The MESs that are pointed by red arrows and dots emerge as $\left|\tilde{\psi}_{L, R}^{0}\right\rangle=\frac{1}{\sqrt{2}}\left(\left|\psi_{1}^{0}\right\rangle \pm i\left|\psi_{2}^{0}\right\rangle\right)$. The MES $\left|\tilde{\psi}_{R(L)}^{0}\right\rangle$ has uniform counterclockwise (clockwise) chiral order for each triangle as illustrated in Fig. 1(b). Furthermore, if we initiate the DMRG state as a complex state, we automatically find such a MES, which spontaneously breaks TRS. The results are demonstrated for $J^{\prime}=0.5$ for a $N=3 \times 24 \times 6$ cylinder, and near identical results are obtained for different parameters in the CSL phase.

The energy and entanglement spectra doubling are signatures of finding the maximally entangled states in each sector, which is forced by the TRS of the system Hamiltonian (here we used a real number initial wavefunction in DMRG calculations which forbids any spontaneous TRS breaking). To demonstrate the nature of the new quantum phase, we first find the minimum entangled states (MESs) in each topological sector ${ }^{28,51,52}$, which represent the eigenstates of the Wilson-loop (string-like) operators encircling the cylinder and are the simplest states of the quasiparticles. In Fig. 3(c), we show two MESs emerging (labeled by two red dots) in the vacuum sector: $\left|\tilde{\psi}_{L(R)}^{0}\right\rangle=\frac{1}{\sqrt{2}}\left(\left|\psi_{1}^{0}\right\rangle \pm i\left|\psi_{2}^{0}\right\rangle\right)$, which are equal magnitude superposition of the real states with a phase difference $\pm \pi / 2$. The MES $\left|\tilde{\psi}_{L}^{0}\right\rangle$ breaks the TRS spontaneously and demonstrates a uniform nonzero chirality order for each triangle as illustrated in Fig. 1(b). The chiral order reaches a value around 0.08 comparable to its classical value $1 / 8$. The conjugate state $\left|\tilde{\psi}_{R}^{0}\right\rangle$ as another MES has the opposite sign of chirality. The doubling of the entanglement spectra for the maximum entropy state simply results from the superposition of the MESs with the same entanglement spectra. Consequently, one finds an entanglement entropy difference $\ln 2$ comparing to the MESs as illustrated in Fig. 3(c). Near identical results and two MESs are also found in the topological degenerating $S$-sector. Furthermore, if we initiate the DMRG state with a random complex number state, we automatically find such a MES, which spontaneously breaks TRS.
By obtaining the MES, we find the topological entanglement entropy $\gamma$ consistent with the result $\ln 2 / 2$ of the $v=1 / 2$ Laughlin state $^{53,54}$. The ED calculations further confirm this state on a $N=3 \times$ $4 \times 3$ cluster by extracting modular transformation matrix ${ }^{51,52}$ from the MESs of two noncontractable cuts (see Supplementary Information for more details).

Quantum phase transitions. We use both the chiral-chiral correlation functions and the topological Chern number obtained from inserting flux to identify the quantum phase diagram and transitions in the $J-J^{\prime}$ model. In Fig. 4(a), we compare the chiral correlations $\left\langle\chi_{i} \chi_{j}\right\rangle$ for the states from the two topological sectors with different system widths at $J^{\prime}=0.5$. We find long-range correlations for the states from both topological sectors, which are further enhanced with increasing system width $L_{y}$. To reveal the quantum phase transitions, we show the chiral correlation functions calculated from the ground state of the vacuum sector for different $J^{\prime}$ in Fig. $4(\mathrm{~b}) .\left\langle\chi_{i} \chi_{j}\right\rangle$ is positive everywhere and has the long-range order for $0.1 \leq J^{\prime} \leq 0.7$, while transitions to other phases are detected at $J^{\prime}=0.05$ and 0.8 by identifying the exponential decaying chiral correlations.

In the flux insertion simulations, we find that the Chern number remains to be quantized at $C=1 / 2$ for the same parameter range 0.1 $\leq J^{\prime} \leq 0.7$, thus we establish the quantum phase diagram as shown in Fig. 1(a). The quantum phase transition around $J^{\prime} \sim 0.7-0.8$ is charaterized by an excitation gap closing in the bulk of system, where we detect a strong bulk magnetization (boson density) response to the inserted flux. Between $J^{\prime}=0$ and 0.1 , we detect a strong nonuniform 

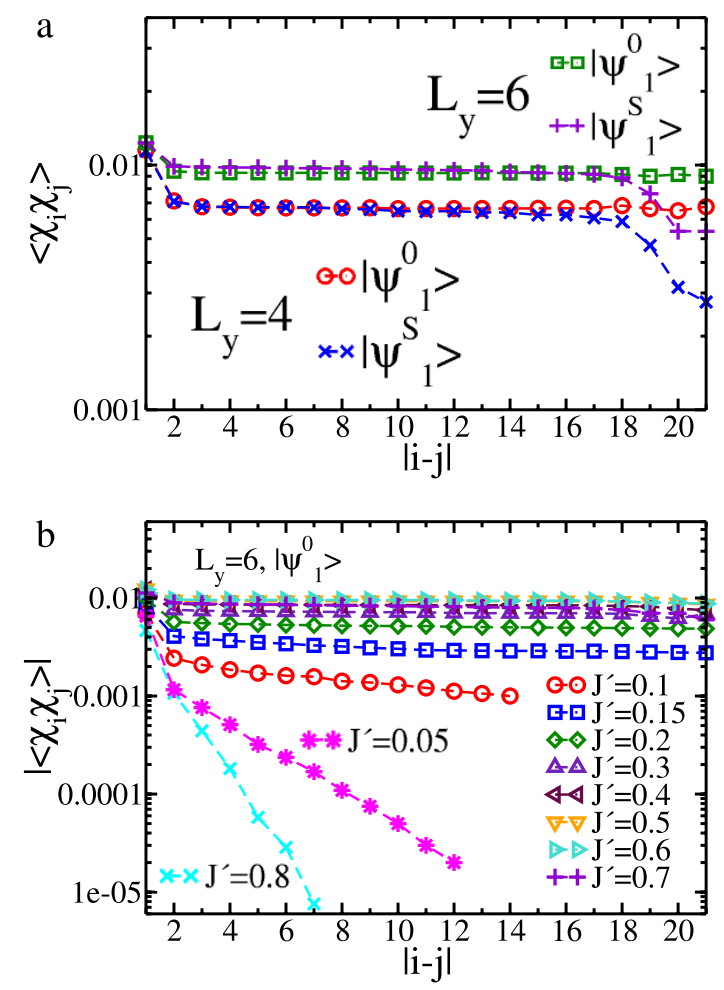

Figure 4 | Long-range chiral-chiral correlation function for CSL. (a) Loglinear plot of the chiral-chiral correlation function $\left\langle\chi_{i} \chi_{j}\right\rangle$ versus the distance of triangles $|i-j|$ along the $x$ direction on the $N=3 \times 24 \times 4$ and $3 \times 24 \times 6$ cylinders for the ground states in both vacuum and S-sectors at $J^{\prime}=0.5$. All the correlations are positive and increase with the system width $L_{y}$. In the S-sector, $\left\langle\chi_{i} \chi_{j}\right\rangle$ have small edge effects due to the presence of two localized spins from pinning. (b) Log-linear plot of absolute correlation $\left|\left\langle\chi_{i} \chi_{j}\right\rangle\right|$ versus $|i-j|$ along the $x$ direction for $N=3 \times 24 \times 6$ cylinders at various $J^{\prime}$ obtained from the ground state in the vacuum sector. All the correlations demonstrate long-range order (and they are also positive) for the intermediate phase $0.1 \leq J^{\prime} \leq 0.7$, while they decay exponentially at $J^{\prime}=0.05$ and 0.8 , where transitions to the $Z_{2}$ spin liquid and VBS phase take place. The correlations are near a constant everywhere in the CSL phase, so we choose $i=3$ and vary $j$ to the end of the cylinder.

Berry curvature resulting from the gauge field in the inserting flux simulations, possibly indicating the forming of new quasiparticles and the emerging of $Z_{2}$ QSL. We also study the stability of the CSL when the second and third neighbor couplings are different. We find the CSL phase in a region around the line with $J_{2}=J_{3}$. For example, when $J_{2}=0.1$, the CSL is robust for $0.1 \lesssim J_{3} \lesssim 0.3$. Physically, the $J_{3}$ coupling suppresses the magnetic order formed in the $J_{1}-J_{2}\left(J_{2} \sim\right.$ 0.2) kagome model, thus substantially enlarges the non-magnetic region. Meanwhile, classically the $J_{3}$ term will enhance a noncoplanar spin chiral order ${ }^{38}$, which may induce a CSL in the quantum $J_{1}-J_{2}-$ $J_{3}$ model as demonstrated here.

\section{Discussion}

In the past twenty years, the gapped QSL in realistic magnetic systems have attracted intensive attention. While the $\mathrm{NN}$ or $J_{1}-J_{2}$ $\mathrm{KHM}^{27-30}$ is the primary candidate of a possible $Z_{2}$ QSL, there are still many puzzles left unresolved. The frustrated kagome antiferromagnets Herbertsmithite $\mathrm{Cu}_{3}(\mathrm{Zn}, \mathrm{Mg})(\mathrm{OH})_{6} \mathrm{Cl}_{2}$ and Kapellasite $\mathrm{Cu}_{3} \mathrm{Zn}(\mathrm{OH})_{6} \mathrm{Cl}_{2}{ }^{18,21-23}$ are possible candidates of QSL; however, they appear to be more consistent with gapless or critical states. At theoretical side, redundant low-energy excitations are found for the NN KHM from ED simulations ${ }^{55}$, variational studies find that $U(1)$ gapless QSL $^{45,56}$ has relatively low energy, and DMRG studies have not been able to identify all the four topological sectors for $Z_{2} \mathrm{QSL}^{49}$. Our finding of the robust CSL at the neighbor of the NN KHM indicates that the latter is not a fully developed $Z_{2}$ QSL yet, and the nature of states for the experimental relevant kagome systems may be strongly affected by a new quantum critical point between two gapped QSLs, the $Z_{2}$ and the CSL. In a parallel work, a CSL has also been uncovered in an anisotropic kagome spin system ${ }^{37}$ with only spin-z interactions for further neighbors. We believe that our numerical findings will stimulate new theoretical and experimental researches in this field to resolve the nature of the quantum phases for different frustrated magnetic systems. An exciting next step will be identifying theoretical models and experimental materials which can host exotic topological superconductivity by doping different CSLs.

\section{Methods}

DMRG is a powerful tool to study the low-lying states of strongly correlated electron systems ${ }^{46}$. The accuracy of DMRG is well controlled by the number of kept states $M$, which denotes the $M$ eigenstates of the reduced density matrix with the largest eigenvalues. The highly efficiency of DMRG for one-dimensional systems or two dimensional cylinder systems have been shown for different systems ${ }^{27,47}$. An improvement in DMRG calculations is to implement symmetry to reduce the Hilbert space. The spin-z or total particle $U(1)$ symmetry is commonly used in DMRG, which is preserved in many model systems. For some systems with spin rotational $S U(2)$ symmetry such as the Heisenberg spin model, the more efficient choice is to apply the $S U(2)$ symmetry $^{57}$, from which we can obtain more accurate results for wider systems. This algorithm has been applied to study various frustrated Heisenberg systems successfully ${ }^{29,58,59}$

Details of the $S U(2)$ DMRG calculation. We study the frustrated KHM without flux using SU(2) DMRG. We study the cylinder system with open boundaries in the $x$ direction and periodic boundary condition in the $y$ direction. For $L_{y}=4\left(L_{y}=6\right)$ systems, we keep up to 3000 (4600) SU(2) states with the DMRG truncation error $\epsilon \simeq 1 \times 10^{-6}\left(\epsilon \simeq 1 \times 10^{-5}\right)$ for most calculations. To find the ground states in both vacuum and S- topological sectors on cylinders in the DMRG calculations, we take pinning sites in the open boundaries or insert flux to target the two different sectors ${ }^{49}$.

Adiabatic DMRG and fractionally quantized Chern number. For the first time, we develop the numerical flux insertion experiment for cylinder systems based on the adiabatical DMRG simulation to detect the topological Chern number ${ }^{34}$ of the bulk system, which uniquely characterizes the CSL as a $v=1 / 2$ FQHE state emergent from the $J-J^{\prime}$ Heisenberg model on kagome lattice. In this simulation, we impose the twist boundary conditions along the $y$ direction by replacing terms

$S_{i}^{+} S_{j}^{-}+$h.c. $\rightarrow e^{i \theta} S_{i}^{+} S_{j}^{-}+$h.c. for all neighboring $(i, j)$ bonds with interactions crossing the $y$-boundary in the Hamiltonian. Starting from a small $\theta \sim 0$, a state with the definite chirality and sign of Chern number will be randomly selected, which remains the same through out the whole adiabatical process of $\theta=0 \rightarrow 4 \pi$. We find states with the opposite Chern numbers $(C= \pm 1 / 2)$ in different runs of the simulations due to spontaneously TRS breaking. A robust excitation gap $\Delta \sim 0.24$ is obtained for $J^{\prime}=0.5$ after we create two spinons $($ at $\theta=2 \pi$ ) at the opposite edges of the cylinder (see Fig. 2(a)), which protects the CSL state. This method can be applied to study different interacting systems and characterize different topological states.

1. Tsui, D. C., Stormer, H. L. \& Gossard, A. C. Two-Dimensional Magnetotransport in the Extreme Quantum Limit. Phys. Rev. Lett. 48, 1559 (1982).

2. Laughlin, R. B. Anomalous Quantum Hall Effect: An Incompressible Quantum Fluid with Fractionally Charged Excitations. Phys. Rev. Lett. 50, 1395 (1983).

3. Prange, R. E. \& Girvin, S. M. The quantum Hall effect (Springer, Berlin, 1987).

4. Wen, X. G. Topological Orders in Rigid States. Int. J. Mod. Phys. B 4, 239 (1990).

5. Halperin, B. I. Statistics of quasiparticles and the hierarchy of fractional quantized Hall states. Phys. Rev. Lett. 52, 1583 (1982).

6. Haldane, F. D. M. Fractional quantization of the Hall effect: a hierarchy of incompressible quantum fluid states. Phys. Rev. Lett. 51, 605 (1983).

7. Wen, X. G. Chiral Luttinger liquid and the edge excitations in the fractional quantum Hall states. Phys. Rev. B 41, 12838 (1990).

8. Wen, X. G. Mean-field theory of spin-liquid states with finite energy gap and topological orders. Phys. Rev. B 44, 2664 (1991).

9. Balents, L. Spin liquids in frustrated magnets. Nature 464, 199 (2010).

10. Anderson, P. W. The Resonating Valence Bond State in $\mathrm{La}_{2} \mathrm{CuO}_{4}$ and Superconductivity. Science 235, 1196 (1987).

11. Rokhsar, D. S. \& Kivelson, S. A. Superconductivity and the quantum hard-core dimer gas. Phys. Rev. Lett. 61, 2376 (1988).

12. Read, N. \& Sachdev, S. Large-N expansion for frustrated quantum antiferromagnets. Phys. Rev. Lett. 66, 1773 (1991).

13. Moessner, R. \& Sondhi, S. L. Resonating Valence Bond Phase in the Triangular Lattice Quantum Dimer Model. Phys. Rev. Lett. 86, 1881 (2001).

14. Senthil, T. \& Fisher, M. P. A. $Z_{2}$ gauge theory of electron fractionalization in strongly correlated systems. Phys. Rev. B 62, 7850 (2000). 
15. Motrunich, O. I. \& Senthil, T. Exotic Order in Simple Models of Bosonic Systems. Phys. Rev. Lett. 89, 277004 (2002).

16. Balents, L., Fisher, M. P. A. \& Girvin, S. M. Fractionalization in an easy-axis Kagome antiferromagnet. Phys. Rev. B 65, 224412 (2002).

17. Kitaev, A. Anyons in an exactly solved model and beyond. Ann. Phys. 321, 2 (2006).

18. Lee, P. A. An End to the Drought of Quantum Spin Liquids. Science 321, 1306 (2008).

19. Lee, P. A., Nagaosa, N. \& Wen, X. G. Doping a Mott insulator: Physics of hightemperature superconductivity. Rev. Mod. Phys. 78, 17 (2006).

20. Xu, C. \& Sachdev, S. Global phase diagrams of frustrated quantum antiferromagnets in two dimensions: Doubled Chern-Simons theory. Phys. Rev. B 79, 064405 (2009).

21. Janson, O., Richter, J. \& Rosner, H. Modified Kagome Physics in the Natural Spin1/2 Kagome Lattice Systems: Kapellasite $\mathrm{Cu}_{3} \mathrm{Zn}(\mathrm{OH})_{6} \mathrm{Cl}_{2}$ and Haydeeite $\mathrm{Cu}_{3} \mathrm{Mg}(\mathrm{OH})_{6} \mathrm{Cl}_{2}$. Phys. Rev. Lett. 101, 106403 (2008).

22. Fåk, B., Kermarrec, E., Messio, L., Bernu, B., Lhuillier, C., Bert, F., Mendels, P., Koteswararao, B., Bouquet, F., Ollivier, J., Hillier, A. D., Amato, A., Colman, R. H. \& Wills, A. S. Kapellasite: A Kagome Quantum Spin Liquid with Competing Interactions. Phys. Rev. Lett. 109, 037208 (2012).

23. Han, T.-H., Helton, J. S., Chu, S., Nocera, D. G., Rodriguez-Rivera, J. A., Broholm, C. \& Lee, Y. S. Fractionalized excitations in the spin-liquid state of a kagomelattice antiferromagnet. Nature 492, 7429 (2012).

24. Shimizu, Y., Miyagawa, K., Kanoda, K., Maesato, M. \& Saito, G. Spin Liquid State in an Organic Mott Insulator with a Triangular Lattice. Phys. Rev. Lett. 91, 107001 (2003).

25. Kurosaki, Y., Shimizu, Y., Miyagawa, K., Kanoda, K. \& Saito, G. Mott Transition from a Spin Liquid to a Fermi Liquid in the Spin-Frustrated Organic Conductor $(\mathrm{ET})_{2} \mathrm{Cu}_{2}(\mathrm{CN})_{3}$. Phys. Rev. Lett. 95, 177001 (2005).

26. Itou, T., Oyamada, A., Maegawa, S., Tamura, M. \& Kato, R. Quantum spin liquid in the spin-1/2 triangular antiferromagnet $\mathrm{EtMe}_{3} \mathrm{Sb}\left[\mathrm{Pd}(\mathrm{dmit})_{2}\right]_{2}$. Phys. Rev. B 77, 104413 (2008)

27. Yan, S., Huse, D. \& White, S. R. Spin-Liquid Ground State of the $S=1 / 2$ Kagome Heisenberg Antiferromagnet. Science 332, 1173 (2011).

28. Jiang, H. C., Wang, Z. \& Balents, L. Identifying Topological Order by Entanglement Entropy. Nat. Phys. 8, 902 (2012)

29. Depenbrock, S., McCulloch, I. P. \& Schollwöck, U. Nature of the Spin Liquid Ground State of the $S=1 / 2$ Kagome Heisenberg Model. Phys. Rev. Lett. 109, 067201 (2012)

30. Jiang, H. C., Weng, Z. Y. \& Sheng, D. N. Density Matrix Renormalization Group Numerical Study of the Kagome Antiferromagnet. Phys. Rev. Lett. 101, 117203 (2008)

31. Kalmeyer, V. \& Laughlin, R. B. Equivalence of the resonating-valence-bond and fractional quantum Hall states. Phys. Rev. Lett. 59, 2095 (1987).

32. Wen, X. G., Wilczek, F. \& Zee, A. Chiral spin states and superconductivity. Phys. Rev. B 39, 11413-11423 (1989).

33. Wilczek, F. Fractional Statistics and Anyon Superconductivity (World Scientific, Singapore, 1990)

34. Haldane, F. D. M. \& Arovas, D. P. Quantized spin currents in two-dimensional chiral magnets. Phys. Rev. B 52, 4223 (1995).

35. Yang, K., Warman, L. K. \& Girvin, S. M. Possible spin-liquid states on the triangular and kagome lattices. Phys. Rev. Lett. 70, 2641 (1993).

36. Yao, H. \& Kivelson, S. A. Exact Chiral Spin Liquid with Non-Abelian Anyons. Phys. Rev. Lett. 99, 247203 (2007).

37. He, Y. C., Sheng, D. N. \& Chen, Y. Chiral spin liquid in a frustrated anisotropic kagome Heisenberg model. Phys. Rev. Lett. 112, 137202 (2014).

38. Messio, L., Bernu, B. \& Lhuillier, C. Kagome Antiferromagnet: A Chiral Topological Spin Liquid? Phys. Rev. Lett. 108, 207204 (2012).

39. Messio, L., Lhuillier, C. \& Misguich, G. Time reversal symmetry breaking chiral spin liquids: Projective symmetry group approach of bosonic mean-field theories. Phys. Rev. B 87, 125127 (2013).

40. Schroeter, D. F., Kapit, E., Thomale, R. \& Greiter, M. Spin Hamiltonian for which the chiral spin liquid is the exact ground state. Phys. Rev. Lett. 99, 097202 (2007).

41. Thomale, R., Kapit, E., Schroeter, D. F. \& Greiter, M. Parent Hamiltonian for the chiral spin liquid. Phys. Rev. B 80, 104406 (2009).

42. Nielsen, A. E. B., Cirac, J. I. \& Sierra, G. Laughlin spin-liquid states on lattices obtained from conformal field theory. Phys. Rev. Lett. 108, 257206 (2012).

43. Bauer, B., Keller, B. P., Dolfi, M., Trebst, S. \& Ludwig, A. W. W. Gapped and gapless spin liquid phases on the Kagome lattice from chiral three-spin interactions. arXiv:1303.6963

44. Hermele, M., Gurarie, V. \& Ana, M. R. Mott Insulators of Ultracold Fermionic Alkaline Earth Atoms: Underconstrained Magnetism and Chiral Spin Liquid. Phys. Rev. Lett. 103, 135301 (2009).
45. Iqbal, Y., Poilblanc, D. \& Becca, F. Vanishing spin gap in a competing spin-liquid phase in the kagome Heisenberg antiferromagnet. Phys. Rev. B 89, 020407(R) (2014).

46. White, S. R. Density matrix formulation for quantum renormalization groups. Phys. Rev. Lett. 69, 2863 (1992).

47. Schollwöck, U. The density-matrix renormalization group. Rev. Mod. Phys. 77, 259 (2005).

48. Hatsugai, Y. Chern number and edge states in the integer quantum Hall effect. Phys. Rev. Lett. 71, 3697 (1993).

49. He, Y.-C., Sheng, D. N. \& Chen, Y. Obtaining topological degenerate ground states by the density matrix renormalization group. Phys. Rev. B 89, 075110 (2014).

50. Li, H. \& Haldane, F. D. M. Entanglement spectrum as a generalization of entanglement entropy: Identification of topological order in non-Abelian fractional quantum Hall effect states. Phys. Rev. Lett. 101, 010504 (2008).

51. Zhang, Y., Grover, T., Turner, A., Oshikawa, M. \& Vishwanath, A. Quasiparticle statistics and braiding from ground-state entanglement. Phys. Rev. B 85, 235151 (2012).

52. Zhu, W., Sheng, D. N. \& Haldane, F. D. M. Minimal entangled states and modular matrix for fractional quantum Hall effect in topological flat bands. Phys. Rev. B 88, 035122 (2013).

53. Kitaev, A. \& Preskill, J. Topological Entanglement Entropy. Phys. Rev. Lett. 96, 110404 (2006)

54. Levin, M. \& Wen, X. G. Detecting Topological Order in a Ground State Wave Function. Phys. Rev. Lett. 96, 110405 (2006)

55. Läuchli, A. M., Sudan, J. \& Sorensen, E. S. Ground-state energy and spin gap of spin-1/2 Kagome-Heisenberg antiferromagnetic clusters: Large-scale exact diagonalization results. Phys. Rev. B 83, 212401 (2011).

56. Ran, Y., Hermele, M., Lee, P. A. \& Wen, X.-G. Projected-Wave-Function Study of the Spin-1/2 Heisenberg Model on the Kagome Lattice. Phys. Rev. Lett. 98, 117205 (2007).

57. McCulloch, I. P. \& Gulácsi, M. The non-Abelian density matrix renormalization group algorithm. Europhys. Lett. 57, 852 (2002).

58. Gong, S. S., Sheng, D. N., Motrunich, O. I. \& Fisher, M. P. A. Phase diagram of the spin- $1 / 2 J_{1}-J_{2}$ Heisenberg model on a honeycomb lattice. Phys. Rev. B 88, 165138 (2013).

59. Gong, S. S., Zhu, W., Sheng, D. N., Motrunich, O. I. \& Fisher, M. P. A. Plaquette ordered phase and quantum phase diagram in the spin-1/2 $J_{1}-J_{2}$ square Heisenberg model. Phys. Rev. Lett. 113, 027201 (2014).

\section{Acknowledgments}

We thank Y. C. He for extensive discussions. We also thank Leon Balents, Matthew P. A Fisher, Olexei I. Motrunich and F. Duncan M. Haldane for stimulating discussions and explanations of spin liquid as well as topological physics. This research is supported by the National Science Foundation through grants DMR-1205734 (S.S.G.), DMR-0906816 and DMR-1408560 (D.N.S.), the U.S. Department of Energy, Office of Basic Energy Sciences under grant No. DE-FG02-06ER46305 (W.Z.)

\section{Author contributions}

S.S.G. and W.Z. performed main calculations based on different numerical programs they developed. S.S.G., W.Z. and D.N.S. made significant contributions from the design of the project to the finish of the manuscript

\section{Additional information}

Supplementary information accompanies this paper at http://www.nature.com/ scientificreports

Competing financial interests: The authors declare no competing financial interests.

How to cite this article: Gong, S.-S., Zhu, W. \& Sheng, D.N. Emergent Chiral Spin Liquid: Fractional Quantum Hall Effect in a Kagome Heisenberg Model. Sci. Rep. 4, 6317; DOI:10.1038/srep06317 (2014)

This work is licensed under a Creative Commons Attribution-NonCommercialNoDerivs 4.0 International License. The images or other third party material in this article are included in the article's Creative Commons license, unless indicated otherwise in the credit line; if the material is not included under the Creative Commons license, users will need to obtain permission from the license holder in order to reproduce the material. To view a copy of this license, visit http:// creativecommons.org/licenses/by-nc-nd/4.0/ 